\title{
Circulating LncRNAs as Novel, Non- Invasive Biomarkers for Prenatal Detection of Fetal Congenital Heart Defects
}

\author{
Meng Gu Aibin Zheng ${ }^{a}$ Wenjuan Tu ${ }^{\mathrm{a}}$ Jing Zhao ${ }^{\mathrm{a}}$ Lin $\mathrm{Li}^{\mathrm{a}}$ Mengmeng Lib \\ Shuping Han ${ }^{\mathrm{b}}$ Xiaoshan $\mathrm{Hu}^{\mathrm{b}}$ Jingai Zhu ${ }^{\mathrm{b}}$ Ya Pan ${ }^{\mathrm{b}}$ Jun $\mathrm{Xu}^{\mathrm{a}}$ Zhangbin $\mathrm{Yu}^{\mathrm{b}}$ \\ aDepartment of Pediatrics, Changzhou Children's Hospital of Nantong Medical University, Changzhou, \\ bDepartment of Pediatrics, Nanjing Maternity and Child Health Care Hospital of Nanjing Medical \\ University, Nanjing, China
}

\section{Key Words}

Maternal plasma $\cdot$ Long non-coding RNA $\cdot$ Congenital heart defects $\cdot$ Gene ontology analysis - Signal pathway • Biomarker

\begin{abstract}
Objectives: To explore the clinical value of circulating long non-coding RNAs (IncRNAs) as biomarkers to predict fetal congenital heart defects (CHD) in pregnant women. Methods: Differential expression of IncRNAs isolated from the plasma of pregnant women with typical fetal CHD or healthy controls was analyzed by microarray. Gene ontology (GO), pathway and network analysis were performed to study the function of the IncRNAs. Differentially expressed IncRNAs were validated in plasma samples from 62 pregnant women with typical CHD and 62 matched controls by RT-PCR. The sensitivity and specificity of each IncRNA in the diagnosis of fetal CHD was determined by ROC curve analysis. Results: Microarray analysis identified 3694 up-regulated and 3919 down-regulated (fold change $\geq 2.0$ ) IncRNAs. The top ten significantly differentially expressed, CHD-associated IncRNAs were validated by RT-PCR. Five significantly up-regulated or down-regulated IncRNAs were identified: ENST00000436681, ENST00000422826, AA584040, AA709223 and BX478947 with the AUC of ROC curves calculated as $0.892,0.817,0.755,0.882$ and 0.886 , respectively. Conclusions: Specific IncRNAs aberrantly expressed in the plasma of pregnant women with typical fetal $\mathrm{CHD}$ may play a key role in the development of $\mathrm{CHD}$ and may be used as novel biomarkers for prenatal diagnosis of fetal CHD.

\section{Introduction}

Congenital heart defects (CHDs), are the most common type of congenital malformation, accounting for approximately $28 \%$ of all cases. CHDs include structural abnormalities, including the failure of channels to close or improper development of the heart and large

M. Gu and A. Zheng contributed equally to this work.

Professor Jun $\mathrm{Xu}$,
and Professor Zhangbin Yu

KARGER
Department of Pediatrics, Changzhou Children's Hospital of Nantong Medical University, No. 468, Yanling East Road, Changzhou 213003,( China), and Department of Pediatrics, Nanjing Maternity and Child Health Care Hospital of Nanjing Medical University, No.123 Tian Fei Xiang, Mo Chou Road, Nanjing 210004, (China)

E-Mail yyxujun0519@qq.com (Jun Xu) and E-Mail zhangbinyu@njmu.edu.cn (Z Yu) 
blood vessels during embryonic development. The main clinical manifestations include ventricular septal defects (VSD), atrial septal defects (ASDs) and tetralogy of Fallot (TOF) [1, 2] The prevalence of neonatal CHD is approximately $8 \%$, with a perinatal mortality rate as high as $40 \%$ and mortality rate as high as $20 \%$ in the first month following birth [3]. Fetal mortality rates included deaths from pulmonary hypertension, bacterial endocarditis and congestive heart failure, although complex CHD was the main cause of early death [4]. Early diagnosis of CHD is associated with an improved prognosis. Therefore, prenatal detection of fetal CHD is the key to reduce mortality and improve the prognosis of CHD.

The rate of misdiagnosis in routine antenatal care of cardiac abnormalities is very high, although fetal ultrasound echocardiography can be used as a diagnostic tool for CHD. The diagnostic efficiency is only 6-35\%. In addition, results vary significant between testing centers due to a lack of standards for ultrasonic inspection, differences in the quality of ultrasonic diagnostic equipment, the experience of the operator, and policies and guidelines at different centers which may influence the accuracy of ultrasonic diagnosis of fetal CHD. Recently, biomarkers such as increased acylated ghrelin [5], miR-19a [6] and beta human chorionic gonadotropin (HCG $\beta$ ) [7], or decreased activity of BMP4 [8] and low levels of pregnancy associated plasma protein A (PAPP-A) [7] were found to be associated with fetal CHD, although their use as biomarkers for prenatal screening of fetal CHD is not specific.

LncRNAs are a type of RNA with a length of approximately 200 nucleotides that are not translated. LncRNAs are stable in plasma and urine, and show disease and tissue specificity. To date, more than 1000 lncRNAs have been confirmed to be involved in various biological processes, including cell growth, differentiation, cell proliferation and apoptosis $[9,10]$. More and more studies suggest that circulating plasma lncRNAs have great potential as new diagnostic and prognostic biomarkers and play important roles in effective evaluation of treatment in disease such as cardiovascular disease and cancer $[11,12]$. The role of lncRNAs in heart development has been described. Specific fetal CHD-related lncRNAs can be found in the placental tissue in fetal CHD, which suggests that placenta derived IncRNAs may be detected in the peripheral blood of pregnant women.

We hypothesize that circulating lncRNAs in pregnant women can be used as candidate biomarkers for predicting fetal CHD in early pregnancy. In the current study, maternal circulating IncRNAs were screened systematically by Arrarystar IncRNA microarray and verified by RT-PCR using a large number of samples. At the same time, the clinical value of lncRNAs in the early diagnosis of fetal CHD were evaluated.

\section{Materials and Methods}

Study design

This study was approved by the hospital ethics committee. Each participant provided written informed consent.

\section{Study participants}

In this study, paired cases were used to confirm the predictive effects of circulating lncRNAs from pregnant women in the diagnosis of fetal CHD. Between March, 2015 and September, 2015, 152 out-patient cases were collected at the Nanjing Maternity and Child Care Center. Twenty-five cases with a history of heart or cardiovascular disease, pregnancy complications or multiple pregnancies were excluded. In addition, three cases that were later diagnosed with Down's syndrome were also excluded. In total, 124 cases were included in the study, including 62 cases of diagnosed fetal CHD as the disease group and 62 cases as healthy controls. The disease group included 30 cases of VSD, 18 cases of ASD and 14 cases of TOF, which were confirmed by fetal echocardiography. The ages and gestational ages between the two groups were similar in order to reduce error associated with heterogeneity between the groups.

Study stages

The study was divided into two stages. In the first stage, the lncRNAs from the circulating plasma of three pregnant women with typical fetal CHD and three healthy controls of similar age and gestational 


\section{Cellular Physiology Cell Physiol Biochem 2016;38:1459-1471 \begin{tabular}{l|l|l} 
and BOI: 10.1159/000443088 & $\begin{array}{l}\text { C } 2016 \text { The Author(s). Published by S. Karger AG, Basel } \\
\text { www.karger.com/cpb }\end{array}$
\end{tabular} \\ Gu et al.: Biomarkers Screening for CHD Among Circulating LncRNAs}

age were screened using Arraystar microarray. Gene ontology (GO) analysis and signal pathway analysis were performed. By comparing the relative expression levels of lncRNAs in the two groups of pregnant women, we identified IncRNAs potentially involved in CHD based on biological information such as target genes. From there, we designed the corresponding primers for validation in the second stage. In the second stage, differential expression of lncRNAs was screened in pregnant women with typical fetal CHD and six healthy controls of similar age and gestational age. The top ten significantly up-regulated or downregulated IncRNAs were chosen for further large scale sample validation. Finally, the diagnostic sensitivity and specificity of the top five significantly up-regulated or down-regulated lncRNAs were evaluated using ROC curve.

\section{Plasma isolation and RNA extraction}

Five milliliters of EDTA-coagulated blood from each patient was collected and centrifuged with a speed of $4000 \mathrm{rpm}$ for $10 \mathrm{~min}$. The plasma was stored at $-80{ }^{\circ} \mathrm{C}$ in $1.5 \mathrm{~mL}$ RNase-free microcentrifuge tubes for later use.

Total RNA, including IncRNAs, was extracted from $400 \mu \mathrm{L}$ of plasma using the TRIZOL LS kit (Life Technologies, USA) according to the manufacturer's instruction. The purity and concentration of the total RNA was determined using a NanoDrop2000 (Thermo Scientific, USA).Total RNA was reverse transcribed into cDNA with a RevertAid First Strand cDNA Synthesis Kit (Thermo Scientific, USA). The reaction was performed in a $60 \mu \mathrm{L}$ volume containing $1.5 \mu \mathrm{g}$ total RNA, $6 \mu \mathrm{L}$ of $10 \mathrm{mM}$ dNTP mixture, $3 \mu \mathrm{L}$ of the Multiscribe RT enzyme, $12 \mu \mathrm{L}$ of $5 \mathrm{X}$ RT buffer, $3 \mu \mathrm{L}$ RNase inhibitor, $3 \mu \mathrm{L}$ random primers and $33 \mu \mathrm{L}$ water. The reverse transcription program was as follows: $42^{\circ} \mathrm{C}$ for $60 \mathrm{~min}, 70^{\circ} \mathrm{C}$ for $5 \mathrm{~min}$ and $4^{\circ} \mathrm{C}$ hold. The cDNA was preserved at $-80^{\circ} \mathrm{C}$ for later use. The handling and storage of samples was identical to reduce potential intra- and inter-assay error.

\section{Arraystar microarray}

The Arraystar LncRNA gene chip is capable of detecting 30586 lncRNAs and 26109 protein encoding transcripts. LncRNAs are selected from public transcription databases (Refseq, UCSC knowngenes, Gencode) or papers with a high impact factor. At the initial stages of the experiment, the lncRNAs in the circulating plasma of three pregnant women with typical fetal CHD and three matched healthy controls were screened using the Arraystar gene chip detection system. Briefly, an equal amount of RNA from each sample was transcribed to cRNA. Sample labeling and array hybridization were carried out following protocols provided for the Arraystar RNA Flash Labeling Kit and Agilent SureHyb. The washed chip was scanned using an Agilent DNA Microarray Scanner and raw data extracted using the Agilent Feature Extraction software (v11.0.1.1). The original data standardization and subsequent data processing was performed using GeneSpring GX v12.1 Software (Agilent Technologies). A high quality probe was used to screen the original data (at least one of the probes was labeled as Present or Marginal). Differentially expressed lncRNAs between the two groups were screened using p-value/FDP and the differential expression of lncRNAs between two samples were screened through fold change. Finally, we performed GO analysis and signal pathway analysis and selected lncRNAs displaying significant changes to be further examined.

\section{GO analysis}

The GO project provides a controlled vocabulary to describe gene and gene product. The ontology covers three domains: Biological Process, Cellular Component and Molecular Function.Fisher's exact test is used to find whether there is more overlap between the differential expression list and the GO annotation list than would be expected by chance. The p-value denotes the significance of GO terms enrichment in the differential expression genes. The lower the $p$-value, the more significant the GO term(p-value $\leqq 0.05)$.

\section{Quantitative PCR ( $q P C R$ )}

qPCR was performed in triplicate for each plasma sample with GAPDH as an internal reference using Power SYBR Green PCR Master Mix (Life Technologies, USA) in a LifeProFlex PCR detection system (Life Technologies, USA). The reactions were performed in $10 \mu \mathrm{L}$ volumes with $1 \mu \mathrm{L}$ cDNA, $3 \mu \mathrm{L}$ DEPC-treated water, $5 \mu \mathrm{L}$ SYBR green master mix, and $0.5 \mu \mathrm{L}$ each of forward and reverse primers. All primers were designed online and synthesized by Shanghai GENEray Biotech. Primers were validated by amplification of the target gene. The primer sequences and lncRNA IDs are shown in Table 1. The mixture was incubated at 


\section{Cellular Physiology Cell Physiol Biochem 2016;38:1459-1471 \begin{tabular}{l|l} 
and Biochemistry Published online: April 04, 2016 & $\begin{array}{l}\text { D) 2016 The Author(s). Published by S. Karger AG, Basel } \\
\text { www.karger.com/cpb }\end{array}$ \\
\hline
\end{tabular} \\ Gu et al.: Biomarkers Screening for CHD Among Circulating LncRNAs}

$50^{\circ} \mathrm{C}$ for 2 min then real-time PCR performed according to the manufacturer's protocol (Life Technologies, USA). Cycling parameters were as follows: ${ }^{9} 5^{\circ} \mathrm{C}$ for $10 \mathrm{~min}$ for initial denaturation followed by 40 cycles of $95^{\circ} \mathrm{C}$ for $15 \mathrm{sec}$ and $60^{\circ} \mathrm{C}$ for $1 \mathrm{~min}$. The quantitative PCR results were normalized to the reference gene GAPDH. Probe specificity was confirmed by melting curve analysis and gel electrophoresis to identify a single product. The relative expression of target lncRNAs was determined using the comparative cycle threshold (Ct) method $\left(2^{-\Delta \Delta C T}\right)$, where $\Delta \Delta \mathrm{CT}$ was calculated as the mean $\Delta \mathrm{Ct}$ of CHD samples minus the mean $\Delta \mathrm{Ct}$ of controls, where $\Delta \mathrm{Ct}=\mathrm{Ct}_{\text {sample }}-\mathrm{Ct}_{\mathrm{GAPDH}}$.

\section{Statistical Analysis}

All data are presented as means \pm standard deviation (SD) and analyzed using the SPSS21.0 statistical software package (SPSS Inc., USA). The relative expression levels of IncRNAs between the two groups were estimated using Student's t-test where $\mathrm{p}<0.05$ was considered statistically significant and all statistics were two-sided. The efficiency of IncRNAs in the diagnosis of fetal CHD were evaluated with ROC curves. The sensitivity and specificity of each IncRNA was assessed by the area under the ROC curve (AUC) and 95\% confidence interval(CI).

Table 1. Oligonucleotide sequences used in real-time PCR analysis

\begin{tabular}{|c|c|c|c|}
\hline LncRNA ID & Related gene & Forward primer & Reverse primer \\
\hline ENST00000445310 & KCNQ5-IT1 & TACTACССТGCTCСТCСGTG & GAACAAGCCCTGGGGTCTAT \\
\hline NR_034138 & LOC100144602 & TCCTGTTCCTTTGCCTTTCA & СTTCCTTACCTTCGTCCATTTC \\
\hline ENST00000436681 & LINC00281 & GCCTCGGTTTCTCATTTCTT & GTTGCACCTGATTACCTGGAT \\
\hline uc003hws.1 & AK093356 & АATGCTGCСТАСТTCTGCTCT & GGGACCTTGATGGTCTTTTG \\
\hline ENST00000417313 & OSBPL10-AS1 & CCCTTTTGCCTCTTCTGGA & GCAGGAGAATCGCTTGAACT \\
\hline uc001tfa.1 & RMST & CAGTGGGTGACTGATCGTACC & CAACTCCGTGTCCCTTGTGA \\
\hline ENST00000523790 & HOXA-AS4 & GCTGCGGAGAAAGACACGA & GCACGACACTGCCTTCATTAC \\
\hline ENST00000580336 & LINC00470 & CTGGTATCATTTCCTTTCCGTC & GCTGCTCCTACTTTGCTGTTT \\
\hline ENST00000422826 & H19 & CGTTCCAGGCAGAAAGAGC & TGAATTGCGGTGGGTGAG \\
\hline NR_024483 & CECR5-AS1 & GTCCGTACTATCGCCCTGTG & TGATGCTGCCGTTGGTGTC \\
\hline ENST00000413311 & AC093382.1 & GCTGAGAACAGAAAGGAGGACT & GGCGTGTTACAATGAAAGCA \\
\hline ENST00000422353 & AC012668.2 & ССTCCCCAGCTATGCTTCCT & GGGCTTTGCAGATCACTTTT \\
\hline ENST00000436929 & NAALADL2-AS3 & TGCTCTGACAGCCGTGGAA & CATTGGTGGGTTGCCTTTT \\
\hline NR-037608 & SLX1A-SulT1A3 & ACCAACTACACCACCGTCCC & TCTCCGCATAGTCCGCATC \\
\hline NR-046160 & LOC256021 & ATACGGTGGGAAGATGACTGA & TGGACATTGACTGGGTGGC \\
\hline AA584040 & ZNF192P1 & GAGATGGTTTCGCTGGTTCC & CAGGGATTTGTGAGTTGACTGG \\
\hline AA593324 & PMS2L2 & TCTAAGCACTGCGGTGAAGA & CССАСАТССАТTGCСТGA \\
\hline AA709223 & LOC100507463 & CCACCTGAGGAAAGACATCG & AAAGGGTTTGCGGAGAAGT \\
\hline AW449870 & SGOL1-AS1 & CGGAAAGGAGTAAGATGAACAC & TCAATGGAGCAAGGACCAA \\
\hline BG717137 & COL18A1-AS1 & AGAAACCTCCTGCGAATGG & AGCCGTCGGTTGGTGAAA \\
\hline BX478947 & HOXD-AS1 & CCGCAGACTCAGCAGATACTC & GCTTAGATTCAGCCCGATTT \\
\hline $\operatorname{chr} 13: 104871700-104904225+$ & GS1-124K5.4 & GTCACCTGAGCCTGTGATGC & TAAGTGGCGGCGAAAAGT \\
\hline AA938966 & MIR22HG & GTATCTTGTCCTCCGCTTGTG & TGCAGCCTATGAGTCTATCCC \\
\hline AI808306 & BZRAP1-AS1 & TTCATCCCGTTCACCTCCA & CATGACGATCAAGCGAGATTC \\
\hline AK055084R & AK093519 & TGGGCTTTAGTGTCTGAATGTG & AAGCTCCTCAAACAGAACCATG \\
\hline ENST00000547549 & MIR3179-2 & GCAGAAGGGGTGAAATTT & TGGGTTCATTTCTGGGTCTT \\
\hline
\end{tabular}

\section{Results}

\section{Patient Data}

One hundred and twenty-four cases were examined in this study. Sixty-two subjects were pregnant women with fetal CHD while 62 cases were healthy controls. The disease group consisted of 30 cases of VSD, 18 cases of ASD and 14 cases of TOF. All the fetal CHD cases were diagnosed by fetal echocardiography. There were no significant differences in age or gestational weeks between the two groups (Table 2). 
Gu et al.: Biomarkers Screening for CHD Among Circulating LncRNAs $\begin{aligned} & \text { Differentially } \\ & \text { IncRNAs }\end{aligned}$
disease group or specific CHD groups with controls

Expression profiling of IncRNAs identified 17603 differentially expressed lncRNAs in the plasma of pregnant women with typical fetal CHD (Fig. 1). 3694 lncRNAs are significantly up-regulated (fold change $\geq 2$ ) and 3919 lncRNAs are significantly down-regulated in the CHD group.

\begin{tabular}{|c|c|c|c|c|c|c|c|}
\hline \multirow[t]{2}{*}{ Classification } & \multirow[t]{2}{*}{$\mathrm{N}$} & \multicolumn{2}{|c|}{ Age (years) } & \multirow[t]{2}{*}{$\mathrm{p}^{*}$} & \multicolumn{2}{|c|}{ Gestational weeks } & \multirow[t]{2}{*}{$p^{*}$} \\
\hline & & Mean & SD & & Mean & SD & \\
\hline Disease group & 62 & 26.45 & 5.52 & 0.463 & 25.63 & 3.11 & 0.196 \\
\hline Control group & 62 & 25.71 & 3.15 & & 24.06 & 2.39 & \\
\hline \multicolumn{8}{|l|}{ Specific CHD } \\
\hline VSD & 30 & 26.18 & 5.63 & 0.522 & 24.75 & 2.88 & 0.478 \\
\hline ASD & 18 & 25.34 & 3.74 & 0.301 & 26.13 & 3.18 & 0.219 \\
\hline TOF & 14 & 28.47 & 4.89 & 0.577 & 27.52 & 4.06 & 0.892 \\
\hline
\end{tabular}

\section{GO analysis}

GO analysis to describe the IncRNAs in terms of biological processes, cellular components and molecular functions was carried out. Fisher's exact test was used to determine whether there is more overlap between the list of differentially expressed IncRNAs and the GO annotation list than would be expected by chance. The p-value denotes the significance of GO term enrichment in the differentially expressed genes. The lower the p-value, the more significant the GO term $(p<0.05)$. The most highly enriched GOs terms associated with up-regulated transcripts included biological processes, cellular components and molecular functions. The highest

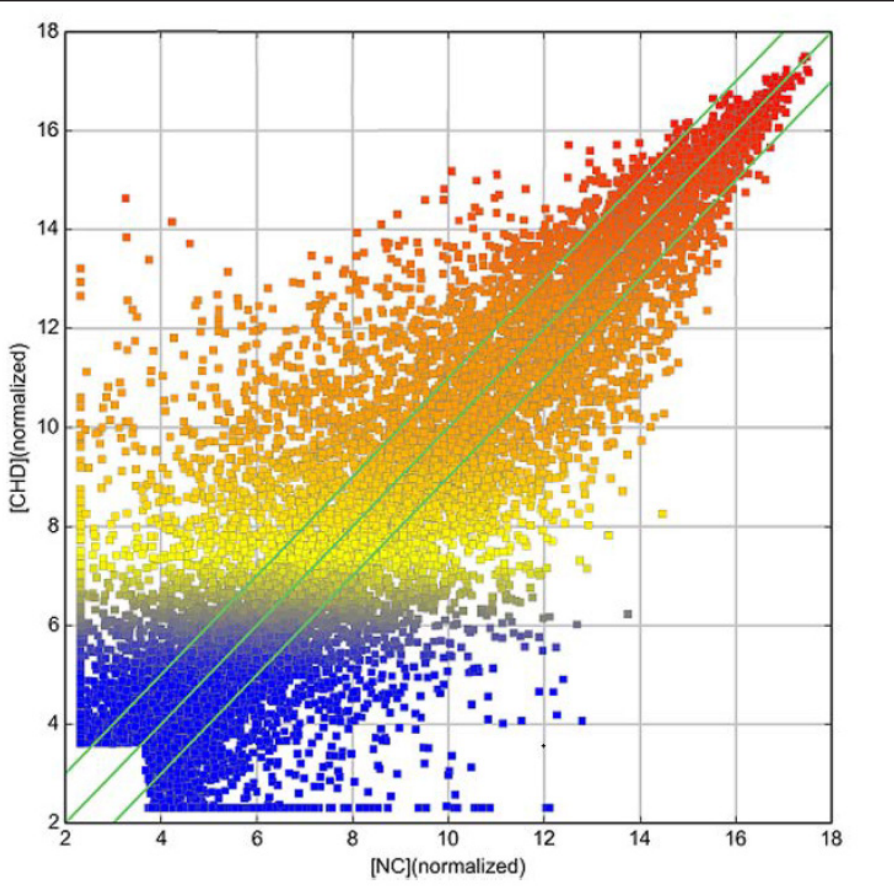

Fig 1. Scatter plot of expression profiles of IncRNAs used to assess reproducibility or variation between chips. enriched GOs target by the down-regulated transcripts were single-organism process (Fig. 2A), organelle (Fig. 2B) and binding (Fig. 2C), while the most highly enriched GOs terms enriched for up-regulated transcripts were regulation of cellular process (Fig. 2D), cell or cell part (Fig. 2E) and binding (Fig. 2F).

\section{Pathway analysis}

The top ten signaling pathways involving down-regulated transcripts are shown in Fig. $3 \mathrm{~A}$. The most highly enriched signal pathway is "hematopoietic cell lineage". The top ten signal pathways involving up-regulated transcripts are shown in Fig. 3B, where the most highly enriched signal pathway is "adherens junction". Amongst these pathways, the RAS signaling pathway has been reported to be involved in hypertrophic cardiomyopathy [13]. The category "morphine addict" has been reported in patients experiencing heart failure [14]. The category "dilated cardiomyopathy" has been associated with patients with ventricular wall stress and cardiac function [15, 16]. The category "Wnt signaling pathway" (Fig. 3C), has been reported in heart valve development. Embryonic myocardial cell proliferation and apoptosis can be promoted through this signaling pathway $[17,18]$.

\section{KARGER}


Fig. 2. GO analysis of differentially expressed lncRNAs which covers three domains: Biological Process, Cellular Component and Molecular Function. (A-C) The GOs most highly enriched in down-regulated transcripts of biological process, cellular component and molecular function. (D-F) The GOs most highly enriched in up-regulated transcripts of biological process, cellular component and molecular function.

\section{Discovery \\ biomarkers}

The aim of this study was to identify lncRNAs in circulating plasma of pregnant women that can serve as biomarkers for predicting fetal CHD. Differential expression of IncRNAs between three pregnant women with fetal CHD and three matched healthy controls were performed using the Arraystar gene chip. The average expression levels and the fold change of IncRNA were analyzed. A comparison of the relative expression of IncRNAs resulted in the identification of 26 IncRNAs closely related to CHD, based on GO analysis. To further assess the 26 lncRNAs, we designed primers to analyze expression. From a

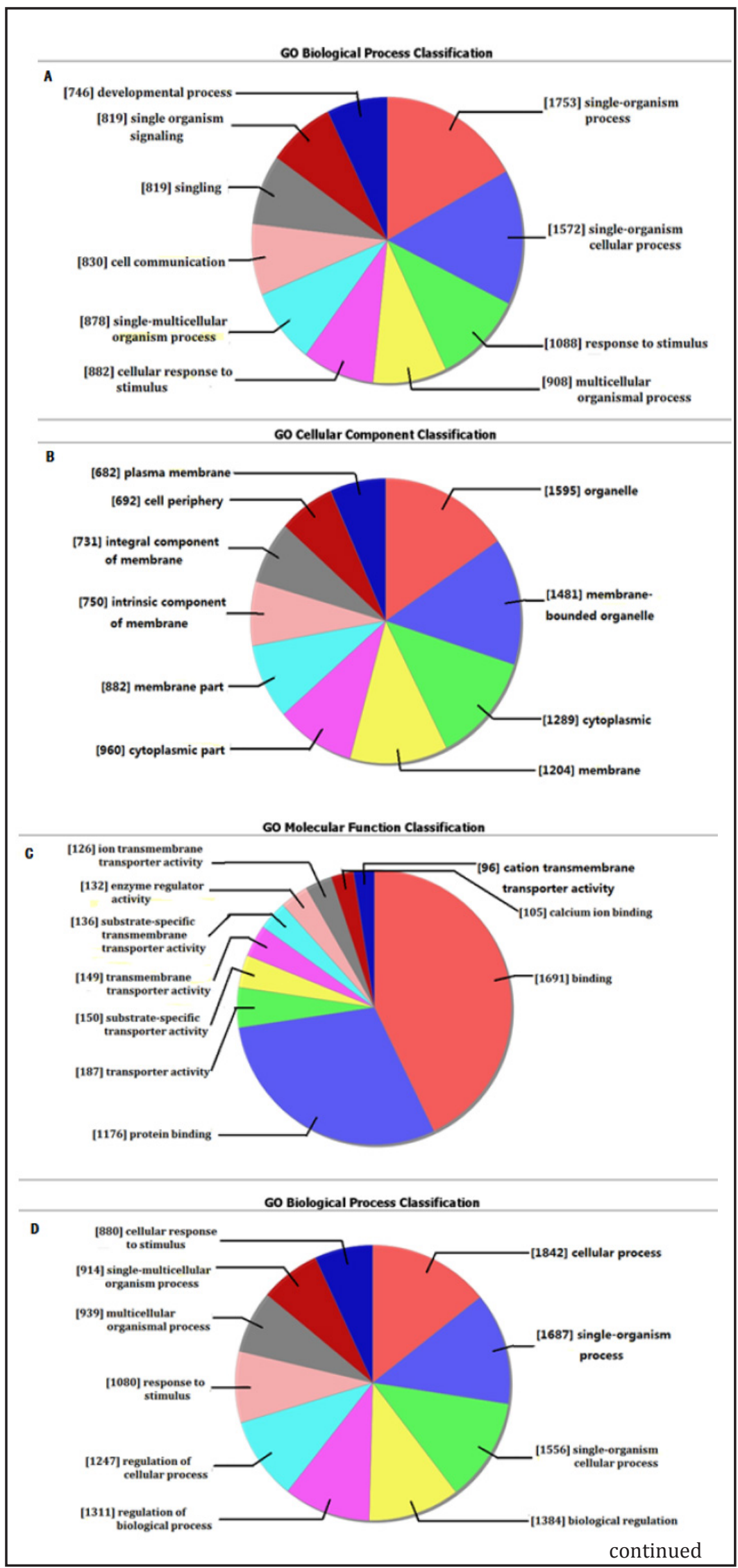




\section{Cellular Physiology Cell Physiol Biochem 2016;38:1459-1471}

\begin{tabular}{ll|l} 
DOI: 10.1159/000443088 & O 2016 The Author(s). Published by S. Karger AG, Basel \\
www kargercom/cpb
\end{tabular}

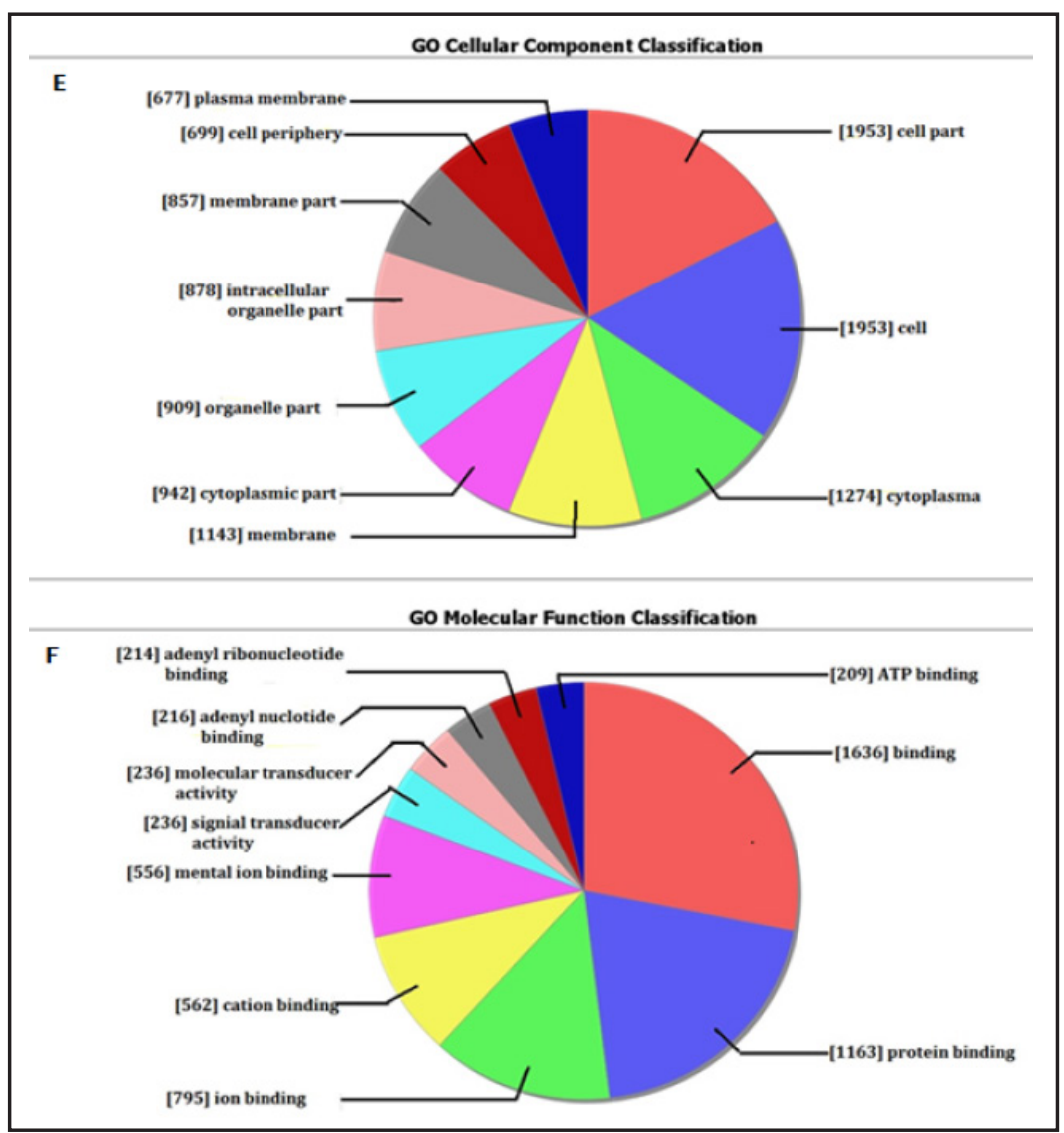

Table 3. LncRNAs identified by Arraystar microarray analysis. *Differential expression of IncRNAs between the two groups was measured by fold-change

\begin{tabular}{llll}
\hline LncRNA ID & Related gene & Fold change* & Regulation \\
\hline ENST00000436681 & LINC00281 & 179.5006428 & up \\
ENST00000422826 & H19 & 41.9889964 & up \\
NR-037608 & SLX1A-SulT1A3 & 332.4933324 & up \\
NR-046160 & LOC256021 & 182.2180039 & up \\
AA584040 & ZNF192P1 & 392.7645742 & down \\
AA709223 & LOC100507463 & 229.9052021 & down \\
BX478947 & HOXD-AS1 & 26.764821 & down \\
chr13:104871700-104904225+ & GS1-124K5.4 & 24.1244643 & down \\
AI808306 & BZRAP1-AS1 & 98.3274756 & down \\
AK055084R & AK093519 & 74.194534 & down \\
\hline
\end{tabular}

preliminary screening of the lncRNAs is six pregnant women with typical fetal CHD and three healthy controls, we identified the ten most highly differentially expressed transcripts. Four up-regulated lncRNAs (ENST00000436681, ENST00000422826, NR-037608 and NR-046160) and six down-regulated IncRNAs (AA584040, AA709223, BX478947, chr13:104871700-104904225+, AI808306 and AK055084R) were chosen for further large sample validation. These results are shown in Table 3.

\section{Validation of IncRNAs}

The ten candidate circulating plasma lncRNAs were validated by RT-PCR with samples obtained from 62 pregnant women with fetal CHD and 62 healthy controls. The quantitative PCR results were normalized to the reference gene GAPDH, and show that five of the ten 


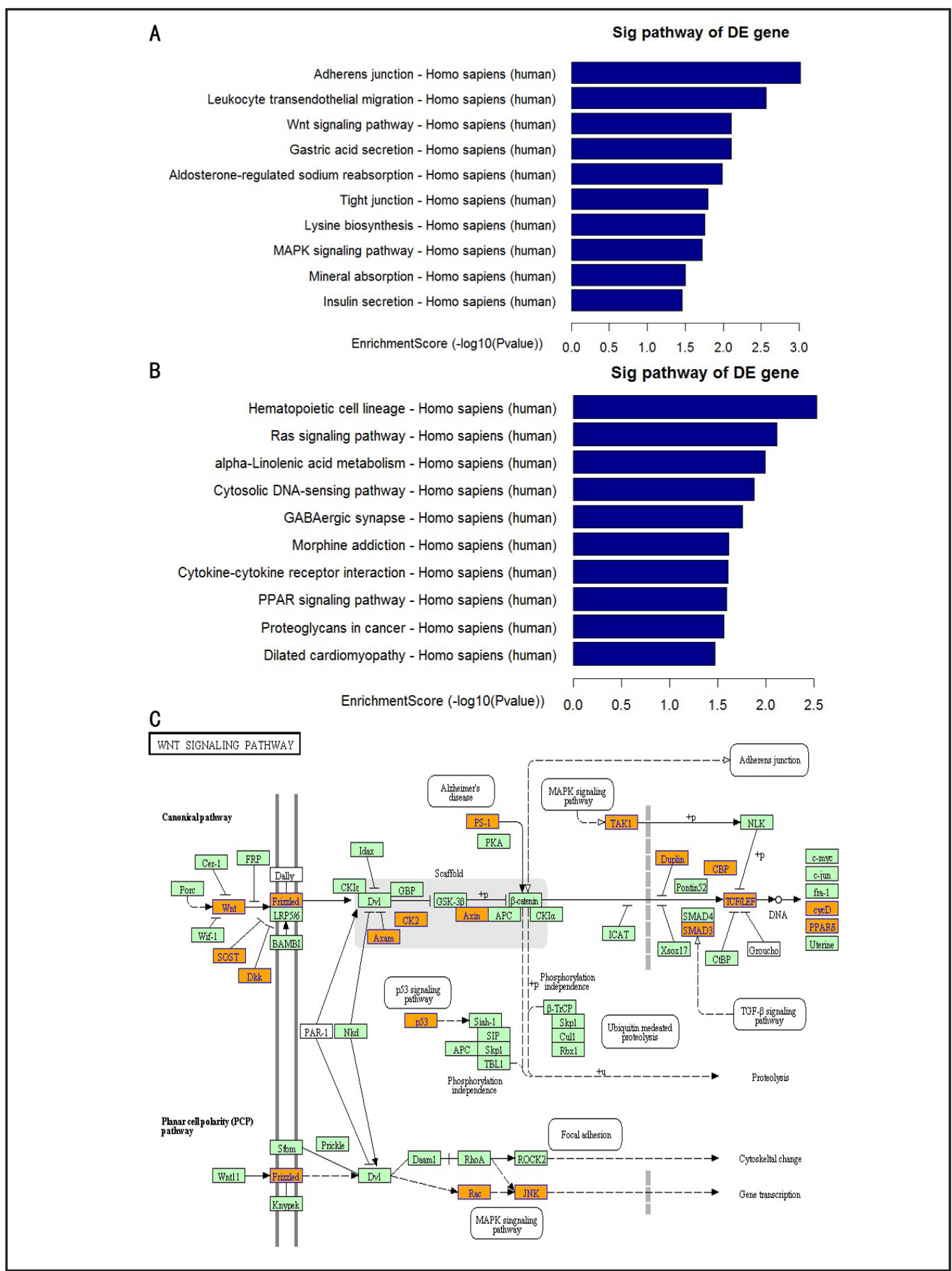

Fig. 3. Pathway analysis is a functional analysis mapping genes to KEGG pathways. EASE score, Fisher $\mathrm{p}$-value or Hypergeometric $\mathrm{p}$-value denote the significance of the pathway correlated to the conditions. The lower the $p$-value, the more significant the pathway $(\mathrm{p}<0.05)$. (A) Pathways corresponding to up-regulated transcripts. (B) Pathways corresponding to down-regulated transcripts. The bar graph in A and B shows the top enrichment score [- $\log 10(\mathrm{p}$-value)] value of the significantly enriched pathway. (C) The schematic diagram of the gene category "Wnt signaling pathway". Nodes marked in yellow are associated with down-regulated genes. Orange marked nodes are associated with up-regulated or only whole dataset genes, while green nodes have no significance. 
A
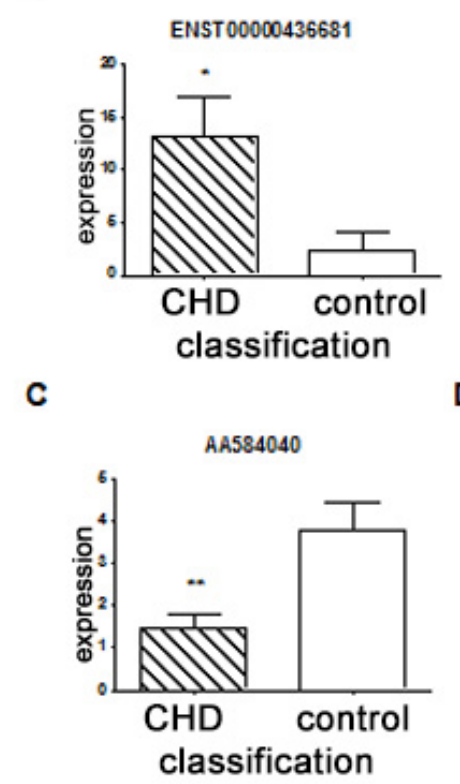

B
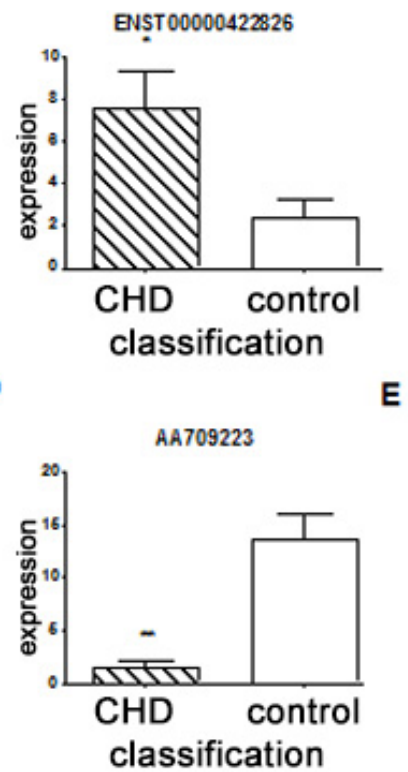

$E$

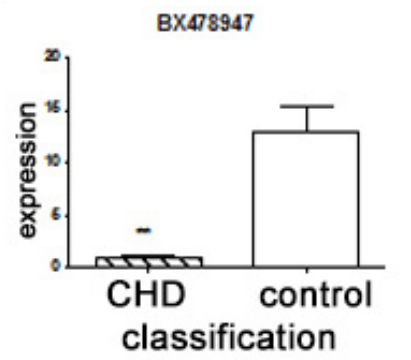

Fig. 4. Relative expression levels of five lncRNAs in 62 pairs of pregnant women with fetal CHD-affected pregnancies and control subjects. (A-E) Histogram of maternal plasma IncRNAs including ENST00000436681, ENST00000422826. AA584040, AA709223 and BX478947. * indicates $\mathrm{p}<0.05$ and ** indicates $\mathrm{p}<0.01$.

Table 4. Differential expression of lncRNAs isolated from maternal plasma between disease and healthy control groups. *Student's t-test. \#The mean of the data was calculated by the comparative cycle threshold $\left(2^{-\Delta \Delta \mathrm{CT}}\right)$ method, in which $\Delta \Delta \mathrm{Ct}$ was calculated by mean $\Delta \mathrm{Ct}$ of CHD samples minus mean $\Delta \mathrm{Ct}$ of healthy controls where $\Delta \mathrm{Ct}=\mathrm{Ct}_{\text {sample }}-\mathrm{Ct}_{\mathrm{GAPDH}}$

\begin{tabular}{lcccccccc}
\hline LncRNA & \multicolumn{3}{c}{ CHD } & \multicolumn{3}{c}{ Control } & Fold change & P* $^{*}$ \\
& $\mathrm{~N}$ & Mean\# & $\mathrm{SD}$ & $\mathrm{N}$ & Mean\# & $\mathrm{SD}$ & & \\
\hline ENST00000436681 & 62 & 13.06 & 29.55 & 62 & 2.43 & 13.78 & 5.37 & $\mathbf{0 . 0 1 1}$ \\
ENST00000422826 & 62 & 7.54 & 14.43 & 62 & 2.40 & 7.19 & 3.14 & $\mathbf{0 . 0 1 3}$ \\
NR-037608 & 62 & 2.08 & 4.61 & 62 & 3.15 & 6.33 & 0.66 & 0.284 \\
NR-046160 & 62 & 1.99 & 4.55 & 62 & 3.44 & 7.56 & 0.58 & 0.198 \\
AA584040 & 62 & 1.47 & 2.72 & 62 & 3.80 & 5.08 & 0.39 & $\mathbf{0 . 0 0 2}$ \\
AA709223 & 62 & 1.55 & 3.61 & 62 & 13.59 & 19.29 & 0.11 & $\mathbf{0 . 0 0 0}$ \\
BX478947 & 62 & 1.01 & 1.23 & 62 & 12.98 & 18.31 & 0.08 & $\mathbf{0 . 0 0 0}$ \\
chr13:104871700-104904225+ & 62 & 0.16 & 0.42 & 62 & 0.07 & 0.20 & 2.29 & 0.118 \\
AI808306 & 62 & 13.22 & 46.25 & 62 & 18.02 & 75.99 & 0.73 & 0.671 \\
AK055084R & 62 & 10.31 & 23.41 & 62 & 14.13 & 27.46 & 0.73 & 0.406 \\
\hline
\end{tabular}

IncRNAs (ENST00000436681, ENST00000422826, AA584040, AA709223 and BX478947) in the disease group were significantly differentially expressed as compared to the healthy controls (p-values are $0.011,0.013,0.002,0.000$ and 0.000 , respectively; Table 4 and Fig. 4), while expression levels of the other five lncRNAs was not significantly different between the two groups (p-values are 0.284, 0.198, 0.118, 0.671 and 0.406; Table 4). As shown in Table 4 , the SD from the mean expression levels of the five differentially expressed lncRNAs is relatively large, which show a high degree of dispersion. Several values of expression levels are very high. A sub-analysis were performed by excluding extreme values and the differences remained significant. VSD, ASD and TOF samples were compared with their matched normal 
Table 5. LncRNA expression in specific CHDs with RNA isolated from maternal plasma. *Student's $\mathrm{t}$-test. \#The mean of the data was calculated by the comparative cycle threshold $\left(2^{-\Delta \Delta \mathrm{Ct}}\right)$ method, in which $\Delta \Delta \mathrm{Ct}$ was calculated by mean $\Delta \mathrm{Ct}$ of CHD samples minus mean $\Delta \mathrm{Ct}$ of healthy controls where $\Delta \mathrm{Ct}=\mathrm{Ct}_{\text {sample }}-\mathrm{Ct}_{\mathrm{GAPDH}}$

\begin{tabular}{lccccccccc}
\hline LncRNA & \multicolumn{3}{c}{ VSD } & \multicolumn{4}{c}{ ASD } & & TOF \\
& $\mathrm{N}$ & Mean\# & $\mathrm{P}^{*}$ & $\mathrm{~N}$ & Mean\# & $\mathrm{P}^{*}$ & $\mathrm{~N}$ & Mean\# & $\mathrm{P}^{*}$ \\
\hline ENST00000436681 & 30 & 9.00 & $\mathbf{0 . 0 3 0}$ & 18 & 21.44 & 0.252 & 14 & 10.99 & $\mathbf{0 . 0 0 3}$ \\
ENST00000422826 & 30 & 3.92 & 0.311 & 18 & 12.86 & 0.171 & 14 & 8.44 & $\mathbf{0 . 0 0 2}$ \\
NR-037608 & 30 & 1.31 & 0.290 & 18 & 2.05 & 0.148 & 14 & 3.76 & 0.666 \\
NR-046160 & 30 & 1.38 & 0.126 & 18 & 1.83 & 0.583 & 14 & 3.52 & 0.142 \\
AA584040 & 30 & 0.86 & $\mathbf{0 . 0 0 5}$ & 18 & 2.43 & 0.237 & 14 & 1.54 & 0.362 \\
AA709223 & 30 & 1.64 & $\mathbf{0 . 0 0 6}$ & 18 & 1.77 & $\mathbf{0 . 0 0 1}$ & 14 & 1.05 & $\mathbf{0 . 0 3 9}$ \\
BX478947 & 30 & 0.65 & $\mathbf{0 . 0 0 3}$ & 18 & 1.54 & $\mathbf{0 . 0 0 2}$ & 14 & 1.08 & $\mathbf{0 . 0 1 6}$ \\
chr13:104871700-104904225+ & 30 & 0.13 & 0.258 & 18 & 0.22 & 0.131 & 14 & 0.17 & 0.859 \\
AI808306 & 30 & 6.81 & 0.552 & 18 & 6.35 & 0.237 & 14 & 35.77 & 0.193 \\
AK055084R & 30 & 5.24 & 0.074 & 18 & 7.30 & 0.225 & 14 & 25.05 & 0.397 \\
\hline
\end{tabular}
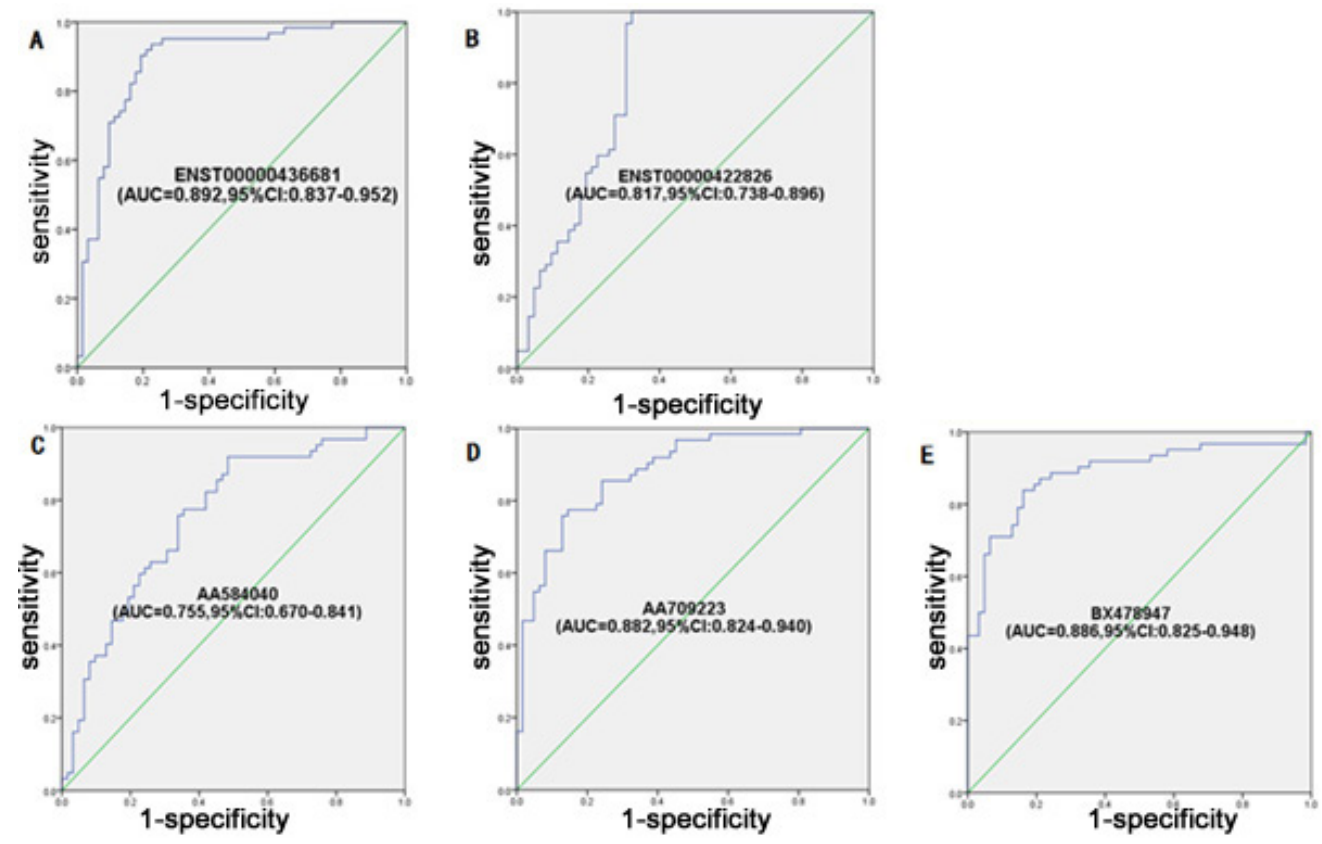

Fig. 5. ROC curve analysis using five IncRNAs to discriminate 62 women with fetal CHD-affected pregnancies from 62 healthy controls. (A) ENST00000436681, (B) ENST00000422826, (C) AA584040, (D) AA709223, (E) BX478947.

controls in order to identify lncRNAs up or down-regulated in these specific defects. There are four significantly changed lncRNAs in VSD (ENST00000436681, AA584040, AA709223 and BX478947) and two in ASD (AA709223 and BX478947). Four IncRNAs show significant differential expression in TOF (ENST00000436681, ENST00000422826, AA709223 and BX478947)( Table 5).

Diagnostic value of maternal plasma lncRNAs for fetal CHD

The sensitivity and specificity of the individual maternal plasma lncRNAs for the prediction of fetal CHD were assessed using ROC curve analysis. The AUC of each lncRNA (ENST00000436681, ENST00000422826, AA584040, AA709223 and BX478947) is shown 
in Fig. 5. The discriminating effect of the five lncRNAs was at the cut-off values of $0.4174,0.7907$, $1.1502,1.9147$ and 1.4227 , respectively. The largest Youden's index (sensitivity + specificity -1) was defined as the optimal diagnostic point. The sensitivity and specificity and AUC of the five lncRNAs are shown in Table 6 and Fig. 5. The results show that effective biomarkers for predicting fetal CHD. five lncRNAs
Table 6. The sensitivity, specificity and AUC of ROC curves of the

\begin{tabular}{lccc}
\hline LncRNA ID & Sensitivity & Specificity & AUC \\
\hline ENST00000436681 & 0.839 & 0.823 & 0.892 \\
ENST00000422826 & 0.710 & 0.710 & 0.817 \\
AA584040 & 0.677 & 0.661 & 0.755 \\
AA709223 & 0.790 & 0.774 & 0.882 \\
BX478947 & 0.839 & 0.823 & 0.886 \\
\hline
\end{tabular}

\section{Discussion}

CHD is one of the most common types of birth defects in the process of embryonic development, with an incidence in newborns of approximately 4\%-5\%. It is the most common non-infective factor in deaths of infants and young children [1]. VSD, accounting for about 25\%-50\% of CHDs, plays an important role in birth defects [19]. To date, there is no gold standard for the diagnosis of fetal CHD, forcing a reliance on fetal echocardiography. Although its diagnostic specificity is high, the rate of misdiagnosis is also quite high.

LncRNAs are a type of non-coding RNA with high tissue and disease specificity. Currently, lncRNAs are a focus of research in tumor and cardiovascular diseases and have become an effective tool for the diagnosis and treatment of cardiovascular diseases and tumors [20,21]. LncRNAs participate in many important regulatory mechanisms such as $\mathrm{X}$-chromosome modification, chromosome silencing, genomic imprinting, transcriptional activation, transcriptional interference and nuclear transport, although they encode little protein [22]. They regulate gene expression at multiple levels such as epigenetic regulation, transcriptional regulation and post transcriptional regulation. Understanding the mechanisms and functional roles of gene regulation is very important in fundamental understanding for cardiac development. Gao et al. [23] reported that the complexity of cardiovascular transcriptome is contributed by RNA editing, RNA splicing and noncoding RNAs. LncRNAs in the heart add intricacy to the regulation network of cardiac gene expression and reveal ways of potential perturbation in response to pathological stressors which demonstrated the potential of these new insights for diagnostic and therapeutic applications. Yang et al. [24] reported that the myocardial transcriptome was regulated in advanced heart failure and lncRNAs markedly altered in response to left ventricular assisted device (LVAD) support which suggest that lncRNAs may play an important functional role in the pathogenesis of heart failure and in reverse remodeling observed with mechanical support.

At present, it is believed that circulating plasma lncRNAs come from cellular apoptosis or necrosis, active release from cells or splitting of circulating cells. Endogenous circulating lncRNAs molecules are not in a free form and often combine with proteins, where they avoid degradation and are more stable so can exist in plasma and urine without becoming targets of enzymatic degradation like other RNA molecules in the blood. They are stable even at room temperature for $24 \mathrm{~h}$ or through repeated freeze-thaw cycles [25]. Li et al. [26] reported expression of lncRNAs in the heart, and whole blood even if normal plasma was negatively associated with their nucleic acid length, whereas the expression in plasma during heart failure was not correlated with length, which suggests that the presence of plasma lncRNAs may not be leakage or a passive response of the heart tissues to heart failure, but instead may be due to active secretion from blood cells or bone marrow hematopoietic stem cells. Therefore, the analysis of IncRNA content in plasma can be used as a non-invasive method for diagnosis of diseases.

\section{KARGER}


High-throughput screening methods, such as IncRNA chip or sequencing make it possible to study the differential expression of lncRNAs in different diseases and provides a convenient platform for subsequent functional studies of lncRNAs or biomarker screening. Abnormalities in transcription, expression, structure and regulation of lncRNAs and their associated binding proteins may be an important factor in the occurrence and development of many important diseases including cancer and cardiovascular diseases [27, 28]. In this study, plasma lncRNAs isolated from pregnant women with typical fetal CHD and normal healthy controls of similar age and gestational age were screened using an Arraystar gene chip and lncRNAs that might be useful in predicting fetal CHD were examined. We identified 17603 expressed IncRNAs, among which 3694 were up-regulated and 3919 were downregulated using abundant and varied probes accounting for 30586 lncRNAs and 26109 protein encoding transcripts in a microarray. The functions of most of these lncRNAs have not been characterized. GO analysis and pathway analysis were carried out and an expression network was constructed to explore the biological functions and potential mechanisms of these differentially expressed lncRNAs in plasma of pregnant women with fetal CHD. The top ten significantly differentially expressed lncRNAs were ENST00000436681, ENST00000422826, NR-037608, NR-046160, AA584040, AA709223, BX478947, chr13:104871700-104904225+, AI808306 and AK055084R. They were validated by RTPCR in all 62 paired cases where we found five to be significantly up-regulated or downregulated (ENST00000436681, ENST00000422826, AA584040, AA709223 and BX478947). Subsequently, we generated ROC curves using SPSS21.0 statistical analysis software. This shows that these five lncRNAs have a high diagnostic value in predicting fetal CHD. We further examined expression of the five lncRNAs in specific CHD subgroups and found that four IncRNAs (ENST00000436681, AA584040, AA709223 and BX478947) were associated with VSD. Two IncRNAs were associated with ASD (AA709223 and BX478947) and four lncRNAs were associated with TOF (ENST00000436681, ENST00000422826, AA709223 and BX478947). To our knowledge, this is the first exploration of the clinical value of lncRNA dynamic changes in pregnant women, which could be applied in disease screening, diagnosis, prognosis and follow-up treatment.

In conclusion, we have identified differentially expressed lncRNAs which have potential diagnostic value in predicting fetal CHD. We have provided evidence that circulating plasma lncRNAs may serve as novel biomarkers for the diagnosis of fetal CHD and provided a solid foundation for further exploration of the pathogenesis of fetal CHD using biomarkers. In a follow-up study, we will expand sample size to further evaluate the value of circulating plasma lncRNAs in the diagnosis of fetal CHD.

\section{Acknowledgements}

This study was supported by grants from the National Natural Science Foundation of China (grant no. 81470376), the National Natural Science Foundation of Jiangsu Province of China (No. BK20141077), and the Nanjing Medical Science and Technique Development Foundation (No. 201402025, YKK14123).

\section{Disclosure Statement}

We declare that there is no conflict of interest regarding the publication of this manuscript.

\section{References}

1 Meberg A, Lindberg H, Thaulow E: Congenital heart defects: the patients who die. Acta Paediatr 2005;94:1060-1065.

2 Trojnarska O, Grajek S, Katarzynski S, Kramer L: Predictors of mortality in adult patients with congenital heart disease. Cardiol J 2009;16:341-347. 


\section{Cellular Physiology Cell Physiol Biochem 2016;38:1459-1471 \begin{tabular}{l|l} 
and Biochemistry Published online: April 04, 2016 & $\begin{array}{l}\text { D } 2016 \text { The Author(s). Published by S. Karger AG, Basel } \\
\text { www.karger.com/cpb }\end{array}$
\end{tabular} \\ Gu et al.: Biomarkers Screening for CHD Among Circulating LncRNAs}

3 Sadeck LS, Azevedo R, Barbato AJ, Calil VM, Latorre Mdo R, Leone CR, Ramos JL: Clinical-epidemiologic indications for echocardiographic assessment in the neonatal period. Value of risk groups. Arq Bras Cardiol 1997;69:301-307.

4 Guo Q Shen J, Liu Y, Pu T, Sun K, Chen S: Exome sequencing identifies a c.148-1G>C mutation of TBX5 in a Holt-Oram family with unusual genotype-phenotype correlations. Cell Physiol Biochem 2015;37:1066-1074.

5 Li G, Xia J, Jia P, Zhao J, Sun Y, Wu C, Liu B: Plasma levels of acylated ghrelin in children with pulmonary hypertension associated with congenital heart disease. Pediatr Cardiol 2015;36:1423-1428.

6 Liu X, Yang L, Wang H, Xu G, Zhu S, Li M, Hu X, Zhu J, Zhu C, Xu J, Han S, Yu Z: Effects of miR-19b knockdown on the cardiac differentiation of P19 mouse embryonic carcinoma cells. Mol Med Rep 2015;11:2504-2512.

7 Qian B, Mo R, Da M, Peng W, Hu Y, Mo X: Common variations in BMP4 confer genetic susceptibility to sporadic congenital heart disease in a Han Chinese population. Pediatr Cardiol 2014;35:1442-1447.

8 Chen X, Chang Y, Cui HY, Ren CC, Yu BY: Study on several ultrasound markers combined maternal serum biochemical markers to screen fetal chromosomal aneuploidy at 11 to $13(+) 6$ weeks of gestation. Zhonghua Fu Chan Ke Za Zhi 2013;48:815-818.

9 Harries LW: Long non-coding RNAs and human disease. Biochem Soc Trans 2012;40:902-906.

10 Liu H, Song G, Zhou L, Hu X, Liu M, Nie J, Lu S, Wu X, Cao Y, Tao L, Chen L, Qian L: Compared analysis of lncRNAs expression profiling in pdk1 gene knockout mice at two time points. Cell Physiol Biochem 2013;32:1497-1508.

11 Tang Q, Ni Z, Xu J, Yu H, Yin P: Three circulating long non-coding RNAs act as biomarkers for predicting NSCLC. Cell Physiol Biochem 2015;37:1002-1009.

12 Li J, Wang X, Tang J, Jiang R, Zhang W, Ji J, Sun B: HULC and Linc00152 act as novel biomarkers in predicting diagnosis of hepatocellular carcinoma. Cell Physiol Biochem 2015;37:687-696.

13 Gelb BD, Tartaglia M: RAS signaling pathway mutations and hypertrophic cardiomyopathy: getting into and out of the thick of it. Clin Invest 2011;121:844-847.

14 Kushner DS, Szanto PB: Heart failure, fever and splenomegaly in a morphine addict. Am Med Assoc 1958;166:2162-2167.

15 Scardulla F, Rinaudo A, Pasta S, Scardulla C: Evaluation of ventricular wall stress and cardiac function in patients with dilated cardiomyopathy. Proc Inst Mech Eng H 2016;230:71-74.

16 Tsuda E, Negishi J, Noritake K, Iwasa T, Abe T: Left ventricular reverse remodeling with infantile dilated cardiomyopathy and pitfalls of carvedilol therapy. J Cardiol 2016;67:147-152.

17 Zhang RR, Gui YH, Wang X: Role of the canonical Wnt signaling pathway in heart valve development. Zhongguo Dang Dai Er Ke Za Zhi 2015;17:757-762.

18 Han SP, Pan Y, Peng YZ, Gu XQ, Chen RH, Guo XR: Folbp1 promotes embryonic myocardial cell proliferation and apoptosis through the WNT signal transduction pathway. Int Mol Med 2009;23:321-330.

19 Pan X, Zheng Z, Hu S, Li S, Wei Y, Zhang Y, Cheng X, Ma K: Mechanisms of pulmonary hypertension related to ventricular septal defect in congenital heart disease. Ann Thorac Surg 2011;92:2215-2220.

20 Gao W, Chan JY, Wong TS: Long non-coding RNA deregulation in tongue squamous cell carcinoma. Biomed Res Int DOI: 10.1155/2014/405860.

21 Liu XH, Sun M, Nie FQ, Ge YB, Zhang EB, Yin DD, Kong R, Xia R, Lu KH, Li JH, De W, Wang KM, Wang ZX: LncRNAs HOTAIR functions as a competing endogenous RNA to regulate HER2 expression by sponging miR-331-3p in gastric cancer. Mol Cancer 2014;13:92.

22 Wilusz JE, Sun woo H, Spector DL: Long noncoding RNAs: functional surprises from the RNA world. Genes Dev 2009;23:1494-1504.

23 Gao C, Wang Y: Transcriptome complexity in cardiac development and diseases--an expanding universe between genome and phenome. Circ J 2014;78:1038-1047.

24 Yang KC, Yamada KA, Patel AY, Topkara VK, George I, Cheema FH, Ewald GA, Mann DL, Nerbonne JM: Deep RNA sequencing reveals dynamic regulation of myocardial noncoding RNAs in failing human heart and remodeling with mechanical circulatory support. Circulation 2014;129:1009-1021.

25 Ge Q, Zhou Y, Lu J, Bai Y, Xie X, Lu Z: miRNA in plasma exosome is stable under different storage conditions. Molecules 2014;19:1568-1575.

26 Li D, Chen G, Yang J, Fan X, Gong Y, Xu G, Cui Q, Geng B: Transcriptome analysis reveals distinct patterns of long noncoding RNAs in heart and plasma of mice with heart failure. PloS One 2013;8:e77938.

27 Wang Y, Li Y, Yang Z, Liu K, Wang D: Genome-wide microarray analysis of long non-coding RNAs in eutopic secretory endometrium with endometriosis. Cell Physiol Biochem 2015;37:2231-2245.

28 Wang Y, Gao S, Liu G, Jia R, Fan D, Feng X: Microarray expression profile analysis of long non-coding RNAs in human gastric cardiac adenocarcinoma. Cell Physiol Biochem 2014;33:1225-1238. 